\title{
Analysis of the Photoacoustic Helmholtz Resonator with Conical-Ended Duct
}

\author{
Mariusz Suchenek
}

\begin{abstract}
The acoustic properties of the photoacoustic Helmholtz cell can be improved by conical modification of the duct ends. The cone profile of the duct ends was studied, and according to the previous work, not all modifications of the parameters provide an increase of the $Q$-factor of the resonator. The aim of this work is to investigate the influence of the conical modification of the duct using a step-approximated model.
\end{abstract}

Keywords-Helmholtz resonator, influence of the duct shape, conical duct model

\section{INTRODUCTION}

$\mathrm{O}$ $\mathrm{NE}$ of the most important advantages of the Helmholtz resonator in photoacoustic measurements is low resonant frequency and small size of the acoustic cavity. The measured photoacoustic amplitude $[1,2]$ is reversely proportional to the volume $V$ and stimulating frequency $f$ and is proportional to the incident light intensity $P$ for the spectral absorption coefficient of the gas $\alpha$.

$$
A \propto \frac{P \cdot \alpha}{V \cdot f}
$$

Minimization of the cell volume gives the opportunity to design a cell with a higher sensitivity and to measure lower concentrations.

Moreover, when the cell is operating at the resonance frequency, the amplitude is proportional to the quality factor. From the point of view of the acoustic design of the cell, it is very important to select the appropriate elements making up the resonator. The sensitivity can be improved by the selection of the microphone and sample volume, duct length and diameter. Moreover, the shape of the duct may also be modified [3, 4], allowing the greater flexibility while designing the whole setup.

\section{HELMHOLTZ RESONATOR WITH CONICAL ENDED DUCT}

Typically a photoacoustic Helmholtz cell consists of two cavities connected with a duct. Proposed modification of the Helmholtz resonator uses the conical profiles at both ends of the duct [3]. The typical structure of the photoacoustic Helmholtz cell is presented in fig. 1 a) and modified in fig. $1 \mathrm{~b}$ ).

The author is with the Institute of Electronic Systems, Warsaw University of Technology, Nowowiejska 15/19, 00-665 Warsaw, Poland (email: M.Suchenek@ise.pw.edu.pl).
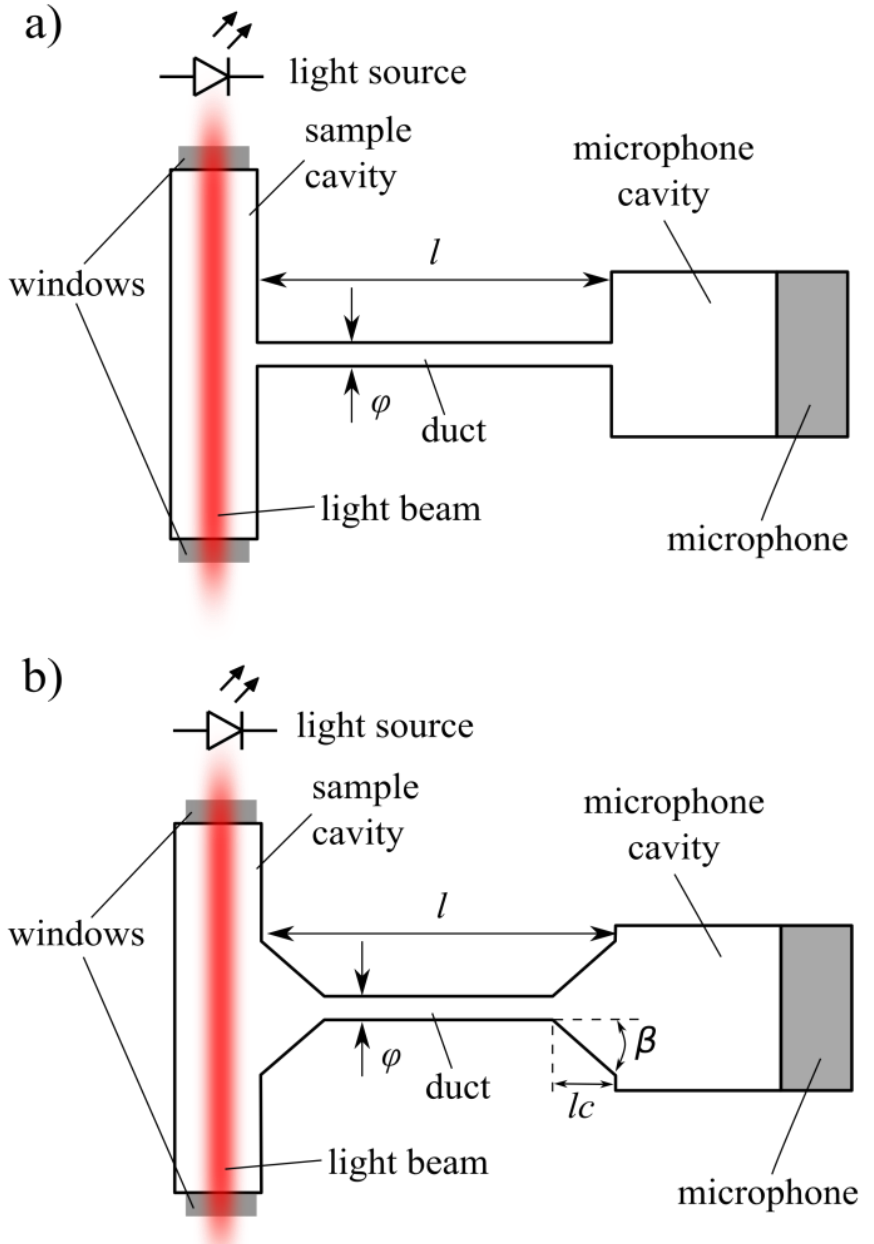

Fig. 1. The structure of the Helmholtz cell used in photoacoustic: a) typical configuration b) modified with cone profiles at the ends of the duct.

Parameters of the photoacoustic cell can be simulated by electroacoustic analogies by lumped element RLC circuits [5 8], transmission line models [6, 8, 9] or by a more complex approach by the FEM simulation $[10,11]$. The simplest model describes the duct in two different ways, by the lumped elements or by the transmission line model. According to the analysis presented in [12] the best results are obtained with transmission line model and model with lumped elements presented by Kastle and Sigrist [13].

The general structure of the model is presented in fig. 2 . The current source corresponds to the light source, capacitance represents the behavior of the sample and microphone cavities. 


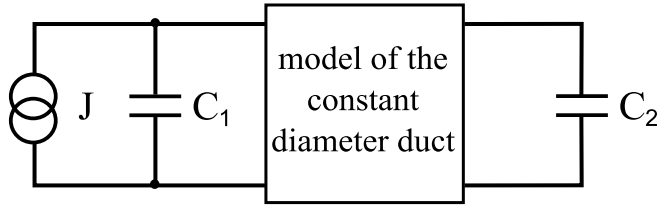

Fig. 2. The general model of the Helmholtz resonator.

The model proposed in [14] extends a photoacoustic model of the Helmholtz resonator that can be found in the literature [5 - 9] by the duct shape. The description of the cone profile is based on the same electro-acoustic analogies as in the lumped element RLC and transmission line models. The section which corresponds to the duct with uniform diameter can be extended on both sides by the section describing the cone profile. The model modified in the described way is presented in fig. 3 .

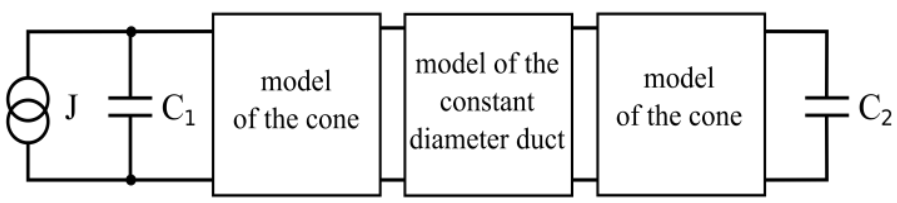

Fig. 3. The model of the Helmholtz resonator with conical ended duct.

Cone sections can be described by the same model as the duct with a uniform diameter, by a serial connection of short sections with uniform diameters. The cone profile divided into six short segments is presented in fig. 4.

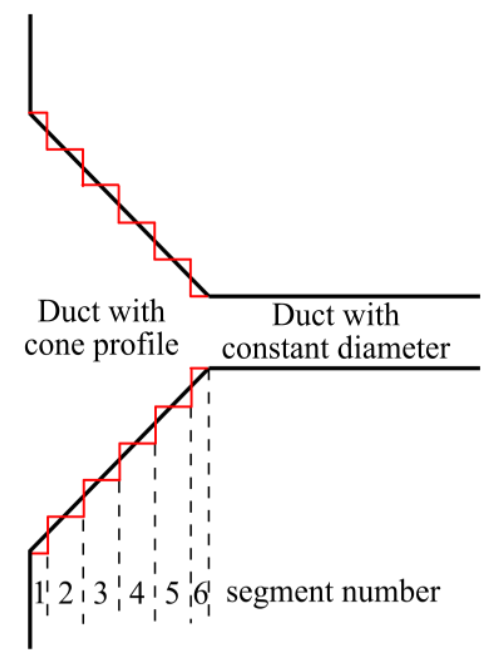

Fig. 4. Cone profile divided into short segments.

Each section is represented as resistance and inductance or transmission line depend on the model [5 - 9]. The accuracy of the approximation depends on the segment length which was also investigated. Based on numerical calculations the influence of segment length on the resonance frequency and Qfactor were determined. The procedure of the method goes as follows the segment length was reduced until the difference between the resonance frequency was less than $1 \mathrm{~Hz}$ and the Q-factor was less than 0.05. As the reference value to calculate the difference was used the cell with the segment length fixed to $2 \mu \mathrm{m}$. On this basis, the segment length was set to $0.11 \mathrm{~mm}$ for the model with lumped elements and to $0.16 \mathrm{~mm}$ for the model with a transmission line. That partly numerical approach gives a quiet good approximation of the cone profile [15 - 17]. Other shapes may be modeled in a similar way. The more precise description of the model including comparison with experimental verification can be found in [14].

\section{NUMERICAL VERIFICATION}

The step-approximated model was used to obtain parameters of Helmholtz resonator with duct ended by the conical shape. From the model two parameters were extracted the Q-factor and the resonant frequency. To evaluate the influence of the conical shape small Helmholtz resonator was chosen, which had $1 \mathrm{~cm}^{3}$ sample and microphone cavity, duct diameter $1 \mathrm{~mm}$ and length $1 \mathrm{~cm}$. Obtained simulations are presented in fig. 5 for the model with the transmission line and fig. 6 for the model with lumped elements.
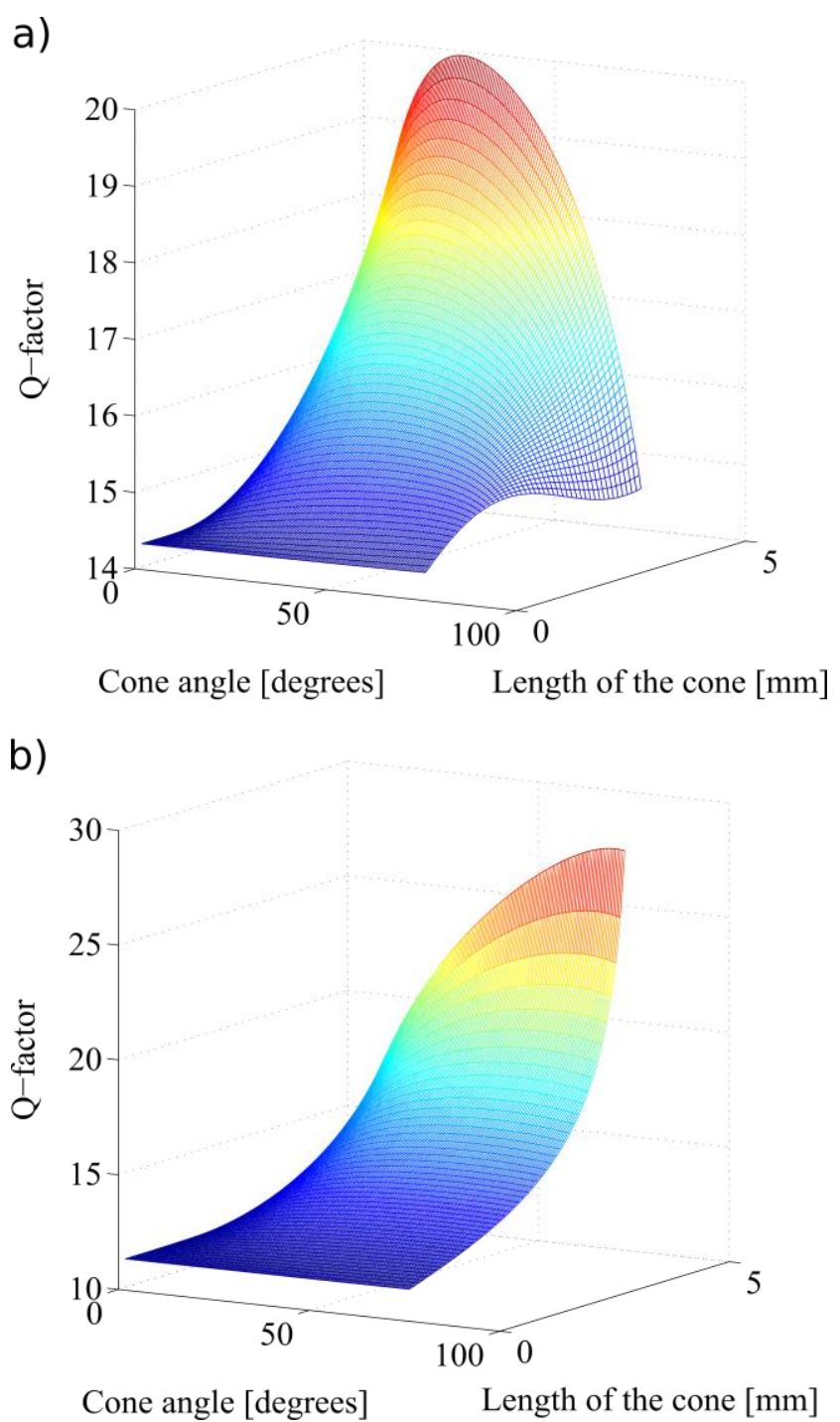

Fig. 5. Influence of the cone angle and length on Q-factor of the Helmholtz resonator with duct ended by the conical shape: a) model with transmission line b) model with lumped elements. 


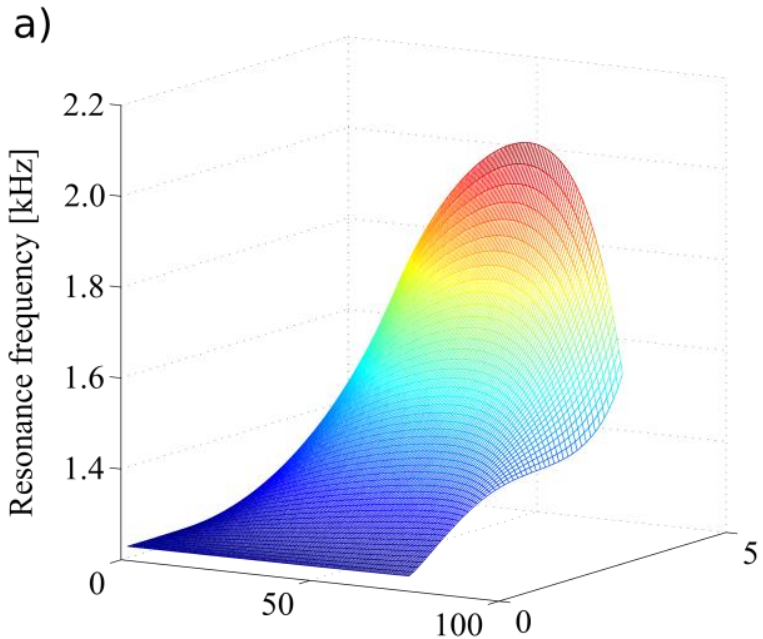

Cone angle [degrees] $\quad$ Length of the cone $[\mathrm{mm}]$

b)

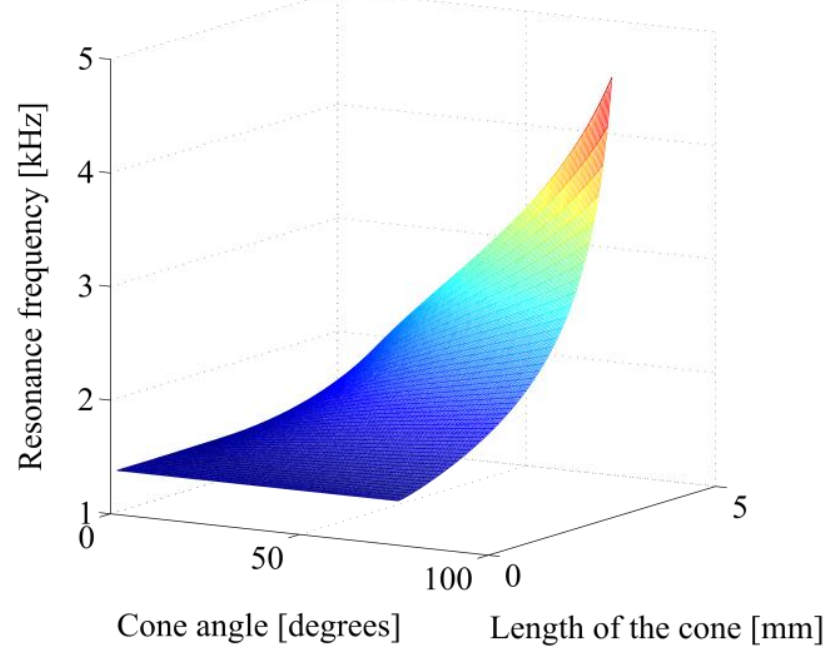

Fig. 6. Influence of the cone angle and length on the resonance frequency of the Helmholtz resonator with duct ended by the conical shape: a) the model with the transmission line b) the model with lumped elements.

Comparing simulation results, one can notice that both models follow the trend, increase length or angle of the cone improves the Q-factor of the cell. Both models agree with this trend. However, the rate of change is different. The model with the transmission line reaches the maximum of the $\mathrm{Q}$-factor for the angle of 28 degrees and the model with the lumped elements at 72 degrees. The difference can be noticed in the resonance frequency as well. The resonance frequency in the model with transmission line reaches the maximum for the angle of 51 degrees while the model with lumped elements does not have such maximum.

In the design of the photoacoustic cell, it is important to increase the quality Q-factor while maintaining the same value of the resonance frequency. Taking into consideration equation (1), the influence of the cone shape on the level of the measured photoacoustic signal amplitude comparing to the amplitude from the unmodified cell is presented in fig. 7 .
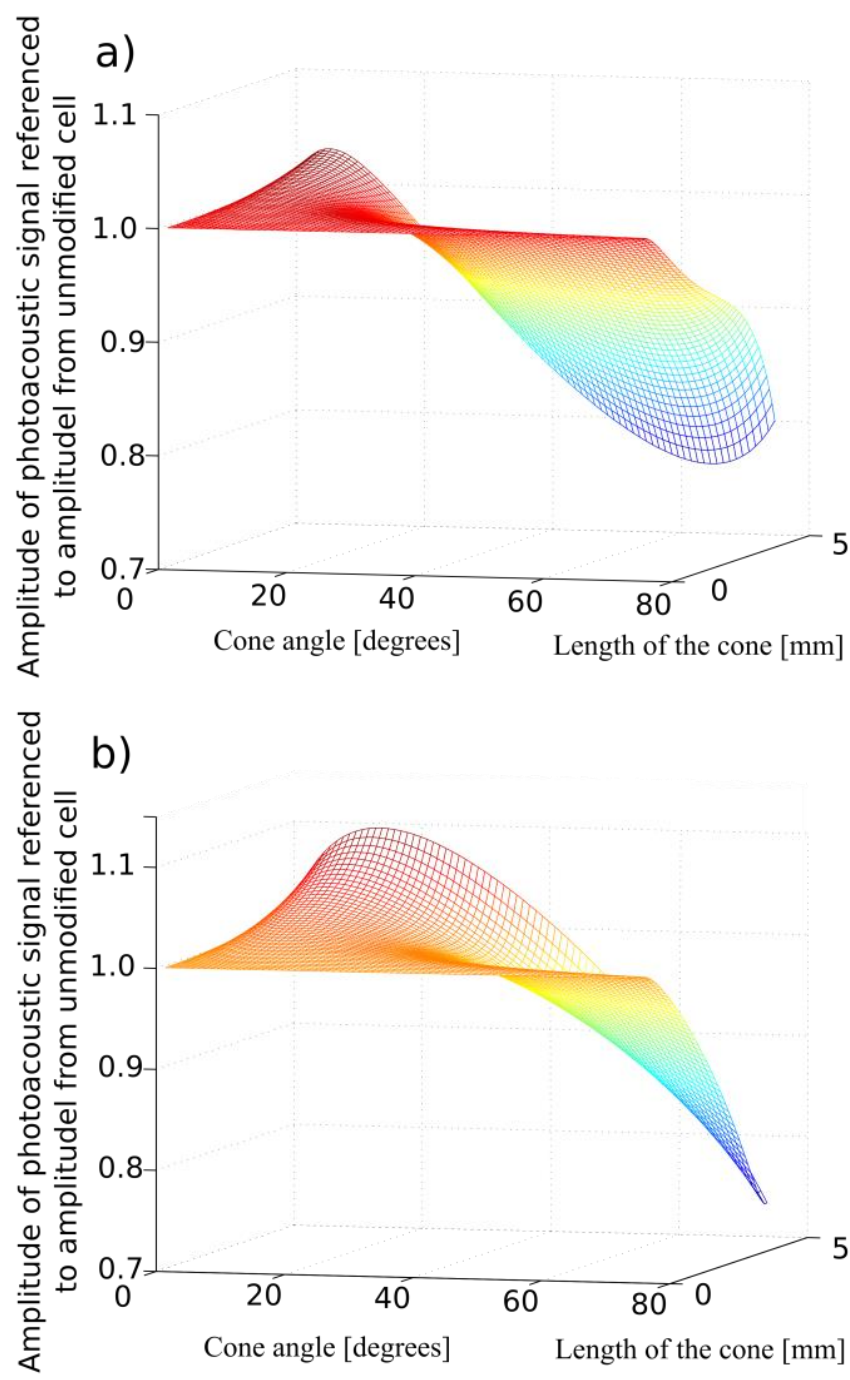

Fig. 7. The ratio of the photoacoustic amplitudes from the modified and the unmodified cells: a) The model with the transmission line b) The model with lumped elements.

The maximum amplitude of photoacoustic signal according to the model with transmission line is achieved for the cone angle of 15 degrees while for the model with lumped elements at 6 degrees. Both models show that further increase in angle will lead to decrease the amplitude.

\section{CONCLUSIONS}

Linear modification of the cone parameter does not result in a linear increase of the resonator Q-factor and resonance frequency. For the given dimensions of the duct and volume cavities, there are optimal values for the length and angle of the cone to achieve the maximum of the Q-factor. However, taking into consideration that the photoacoustic signal is proportional to the Q-factor and inversely proportional to the frequency (for the cell operating at resonance frequency), the maximum for the $\mathrm{Q}$-factor is different from the maximum of the photoacoustic signal. Moreover, the two presented models do not agree on the maximum value of the photoacoustic signal and Q-factor. 


\section{REFERENCES}

[1] R. Bauer, G. Stewart, W. Johnstone, E. Boyd, M. Lengden, "3D-printed miniature gas cell for photoacoustic spectroscopy of trace gases", Opt Lett., vol. 39, iss. 16, 2014, pp. 4796-4799.

[2] A. Miklós, P. Hess, Z. Bozóki, "Application of acoustic resonators in photoacoustic trace gas analysis", Rev. Sci. Instrum.,3 vol. 72, 2001 , pp. 1937-1955.

[3] M. Suchenek, "Model of the photoacoustic Helmholtz resonator with conical-ended duct", Int. J. Thermophys., vol. 35, iss. 12, 2014, pp. 2279 2286.

[4] M. Suchenek, "Influence of the duct shape on the Q-factor of a photoacoustic Helmholtz resonator", Int. J. Thermophys., vol. 32, iss. 4 2011, pp. 886-892.

[5] R. Kastle, M. W. Sigrist, "Temperature-dependent photoacoustic spectroscopy with a Helmholtz resonator", Appl. Phys. B., vol. 63, iss. 4, 1996, pp. 389-397.

[6] O. Nordhaus and J. Pelzl, "Frequency dependence of resonant photoacoustic cells: The extended Helmholtz resonator", Appl. Phys. vol. 25, iss. 3, 1981, pp. 221-229.

[7] M. Mattiello, M. Nikles, S. Schilt, L. Thevenaz, A. Salhi, D. Bart, Y Rouillard, R. Werner, J. Koeth, "Novel Helmholtz-based photoacoustic sensor for trace gas detection at ppm level GaInAsSb/GaAlAsSb DFB lasers", Spectrochemica Acta A, vol. 63, num. 5, 2006, pp. 952-958.

[8] J. Pelzl, K. Klein, O. Nordhaus, "Extended Helmholtz resonator in lowtemperature photoacoustic spectroscopy", Appl. Opt., vol. 21, iss. 1, 1982, pp. 94-99.
[9] T. Starecki, "Loss-improved electroacoustical modeling of small Helmholtz resonators", J. Acoust. Soc. Am., vol. 122, iss. 4, 2007 , pp. 2118-2123.

[10] B. Parvitte, Ch. Risser, R. Vallon, V. Zéninari, "Quantitative simulation of photoacoustic signals using finite element modelling software", Appl. Phys. B, vol 111, iss. 3, 2013 pp. 383-389.

[11] B. Kost, B. Baumann, M. Germer, M. Wolff, M. Rosenkranz, "Numerical shape optimization of photoacoustic resonators", Appl. Phys. B, vol. 102, iss. 1, 2011, pp. 87-93.

[12] M. Suchenek, "Comparison of photoacoustic Helmholtz resonator models”, Proc. SPIE, vol. 6937, 2007, pp. 693710-1 - 693710-8.

[13] R. Kastle, M. W. Sigrist, "Temperature-dependent photoacoustic spectroscopy with a Helmholtz resonator", Appl. Phys. B., vol. 63, iss. 4, 1996, pp. 389-397.

[14] M. Suchenek, "Model of the photoacoustic Helmholtz resonator with conical-ended duct", Int. J. Thermophys., vol. 35, iss. 12, 2014, pp. 2279 2286.

[15] F. J. Young, "Impedance of Tapered Structures", J. Acoust. Soc. Am., vol. 39, no. 5, 1966, pp. 841-846.

[16] F. J. Young, B. H. Young, "Impedance of Tapered Structures", J. Acoust. Soc. Am., vol. 33, no. 9, 1961, pp. 1206-1210.

[17] F. J. Young, B. H. Young, "Smoothly and Step Tapered Structures", J. Acoust. Soc. Am., vol. 33, no. 6, 1961, pp. 813 